\title{
NF- $\kappa$ B, Caspase-3 and p53 Pathways are Involved in Resveratrol-Chitosan \\ Nanoparticles-Induced Apoptosis in Hela Cells with Oxidative Stress
}

\author{
Mohammed A Hussein ${ }^{*}$, Raghad M Mahmmed², Afnan A Sebawaih², \\ Basma A El-Maghraby², Eman E Mohammed ${ }^{2}$, Zekra S Mohamed ${ }^{2}$ and \\ Ali A Ali ${ }^{3}$ \\ ${ }^{1}$ Biochemistry Department, Faculty of Applied Medical Sciences, October 6 University, \\ Sixth of October City, Egypt \\ ${ }^{2}$ Medical Labs Department, Faculty of Applied Medical Sciences, October 6 University, \\ Sixth of October City, Egypt \\ ${ }^{3}$ Vice president of post graduate studies, October 6 University, Sixth of October City, \\ Egypt
}

*Corresponding Author: Mohammed A Hussein, Biochemistry Department, Faculty of Applied Medical Sciences, October 6 University, Sixth of October City, Egypt.
Received: April 07, 2021

Published: April 29, 2021

(C) All rights are reserved by Mohammed A

Hussein., et al.

\begin{abstract}
Background: Resveratrol is a natural polyphenolic phytoalexin commonly found in fruits, grape and red wine. The objective of this study was to prepare RENE for Hela cell anticancer therapy using low chitosan and tripolyphosphate (TPP) method.

Methods: In the present study resveratrol-chitosan nanoparticles was prepared made in the presences of TPP using a phase inversion method. Morphology, particle size and zeta potential of RENE was then characterized. Furthermore, RENE was evaluated for its cytotoxic effect on Hela cells as well as their effect on TBARS, GSH, GST, caspase-3, NF- $\kappa$ B and P53.

Results: RENE was showed spherical shape with mean particle size of around $49.5 \pm 0.05 \mathrm{~nm}$ with (+15.75) negative zeta potential. The treatment of Hela cells with RENE led to a high inhibition in the Hela cell proliferation as concluded by the low $\mathrm{IC}_{50}$ values 31.89 $\mu \mathrm{g} / \mathrm{ml}$. Also, RENE possess apoptotic properties via activation of the TBARS, caspase-3 and P53 protein expression as well as inhibition NF-Kb, GST and GSH levels.

Conclusion: The results presented here may suggest that RENE possess anticancer and apoptotic effects on Hela cell proliferation, and therefore, can be used as new approach of pharmaceutical drugs. Also, the results clearly suggest that NF- $\mathrm{KB}$, is one of the central players in the synergism of RENE and act as a regulator of caspase-3 and P53.

Keywords: Resveratrol; Resveratrol Nanoemulsion (RENE); Chitosan; Hela; NF- $\kappa$ B and Caspase-3
\end{abstract}

\section{Abbreviations}

RENE: Nanoemulsion Resveratrol Nanoemulsion; TPP: Tripolyphosphate; TBARS: Thiobarbituric Acid Reactive Substances; GSH: Reduced Glutathione; GST: Glutathione S Transferase; NF- $\kappa \mathrm{B}: \mathrm{Nu}-$ clear Factor- $\kappa B$; P53: Tumor Protein P53

\section{Introduction}

Today, one of the major public health problems causing death in the world is cancer [1]. The second common cancer among women worldwide is cervical cancer [3]. Crucial genes involved in this pro- cess include APC, K-ras, DCC, p53, c-myc, COX-2, mismatch repair genes, cell adhesion genes [4,5]. Accumulation of alterations during carcinogenesis leads to impairment of normal growth inhibition by increased cell growth and by inhibition of apoptosis, resulting in clonal expansion of tumor cells [5].

Resveratrol is a common polyphenol found in many fruits; grapes and blueberries [6,7]. It has been studied as cardio protective $[8,9]$, anti-cancer $[10,11]$, antioxidant and anti-inflammatory $[12,13]$ effects. 
The mechanism by whom resveratrol has favorable effects would be related to the ability of resveratrol to improve the bioactivity of nitric oxide and/or its inhibitory effects on COX-1 and $N F-\kappa B$ [15] and to block platelet aggregation with the ability of prostaglandins, synthesized with COX-1, to specifically inhibit [16] such as thromboxane A2, which is a powerful cause of platelet aggregation.

Resveratrol was used in vivo testing to determine its anti-inflammatory bowel disease [16-19]. Nevertheless, there are no reports on the effects of RENE in treatment of cervical cancer. In addition, we are reporting an easy way of preparing resveratrol-chitosan nanoparticles and assessing their apoptotic mechanism in cervical cancer cells, in as extension of our interested research program to evaluate the importance of RENE in the medicine [20-24].

\section{Materials and Methods}

\section{Materials}

Resveratrol, 5-fluorouracil, Chitosan medium molecular weight (Sigma-Aaldrich Chemical Co. catalog NO.448877-50G), Triglyceride tridecanoate (Sigma-Aaldrich Chemical Co. catalog NO.T75175G), Soy lecithin (Avanti Polar Lipids Catalog No. 206775), chloroform (HPLC grade), and PBS triglyceride tripalmites (SigmaAaldrich Chemical Co. catalogue NO.T8127-100g.). ATCC American type of culture collection obtained hela cells (human cervical carcinoma). The purchase of Sigma included dimethyl-sulfoxide, violet crystal and blue dye (St. Louis, Mo., USA). Lonza purchased Fetal Bovine serum, DMEM, RPMI-1640, L-glutamine, HEPES buffer solution, gentamycin and 0,25\% trypsin EDTA. Purple Crystal Stain (1\%): It was then made from $0.50(\mathrm{w} / \mathrm{v})$ violet crystal and 50\% methanol and filtered with ddH2O by a filter paper from Whatmann No.1.

\section{Methods}

\section{Preparation of nanoparticles}

The ionotropic gelation process based on the electrostatic interaction between negative and positive charged molecules was used to create resveratrol-chitosan-TPP nanoparticles [17]. Briefly, Chitosan-TPP nanoparticles loaded with resveratrol existed in amino groups in interaction with anionic TPP salt groups. Chitosan stock solution was produced in acidified distilled water at1 mg/
$\mathrm{mL}$, while TPP solution was produced in distilled water at $1 \mathrm{mg} /$ $\mathrm{mL}$. The chitosan (1 mL) supply was first taken away and its volume was changed to $1,5 \mathrm{~mL}$ using distilled water for 10 minutes.

Next $5 \mu \mathrm{L}$ tween 80 were added to the ethanol resveratrol stock $(1 \mathrm{mg} / \mathrm{mL})$. In the chitosan solution resveratrol was then added. Finally, the TPP solution as an emulsified resveratrol-chitosan solution as a cross-connector $(100 \mu \mathrm{L})$ has been added downwards. The solution obtained was removed for 30 minutes and centrifuged for 5 minutes at $4000 \mathrm{~g}$. The supernatant was finally moved to a new tube and kept for later analysis.

\section{Characterization of resveratrol nanoemulsion}

RENE was performed by a pattern of R-25-28 o C with a D8 Advance X-ray difractometer (Bruker - Germany), with nickel (Ni) filtered with $\mathrm{CuK} \alpha(\mathrm{T}=1.54184 \mathrm{~A} 0)$ as an X-ray source. The resveratrol Nanoscope (RENE) had the crystalline nature and grain dimensions.

Nicolet 6700 (Thermo scientific-US) sample infrared spectrum (IR) is recorded. Thermo-gravimetric test (TGA) TGA-50 was used for thermal analysis (Shimadzu, Japan) Morphology and size of RENE were examined by Scanning Electron Microscope (SEM, JSM- 690, JEOL, Inc., Tokyo, Japan) and Field Emission Transmission Electron Microscopy (FETEM, JSM- 2100F, JEOL Inc.) at an accelerating voltage of $15 \mathrm{Kv}$ and $200 \mathrm{Kv}$.

\section{High-performance liquid chromatography}

RENE has been detected with a high performance liquid chromatography (Shimadzu, Tokyo, Japan) system with a C18 reverse phase column and a UV detector, with a flow rate of $1 \mathrm{~mL}$ per minute. The Mobile Phase consisted of $500 \mathrm{~mL}$ of acetic acid, 2,5 mL of methanol and deionized water. The HPLC is used to quantify non-encapsulated and nano-encapsulated resveratrol concentrations. For the calculation of the peak area was used the external standardization method.

\section{Morphology and resveratrol nanoemulsion (RENE) Character- ization}

Resveratrol nanomulsion morphological characteristics were examined using a microscope field Emission (FETEM, JSM-690, JEOL, Inc.) (SEM, JSM-690, JEOL, Inc.), and a $200 \mathrm{kV}$ and 100.000 
magnification accelerating voltage (FETEM, JSM-2100F, JEOL). A drop of nanoparticles has been covered in carbon and is permitted for 24 hours at room temperature. The TEM picture shows that the NLC and CSNLC average diameter is approximately 67 and $98 \mathrm{~nm}$ each and the shape is round. IR spectrum was also recorded using the KBr pellets on the Shimadzu MR 470.

\section{Entrapment efficiency}

RENE (5.0 mg), $5.0 \mathrm{~mL}$ distillated water and $5.0 \mathrm{ml}$ of ethyl acetate, was crushed and dispersed. After the mixture was carefully mixed, the ethyl acetate phase was separated. The UV-Vis spectrophotometer has been used for the free resveratrol content of 419 $\mathrm{nm}$ in the phase of ethyl acetate. Equation calculated for drug entrapment efficiency (1):

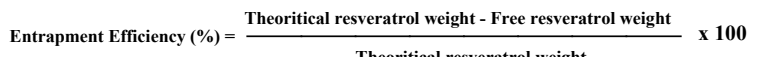

\section{Anticancer assay}

Cytotoxicity evaluation using viability assay

The cells were seeded in the 96-well plate at a cell concentration of 1 to $10^{4}$ cells in a well in a growth medium of $100 \mu \mathrm{l}$ for the cytotoxicity analysis. Fresh medium with various resveratrol nanoemulsion concentrations (RENE) was added after 24 hours of seeding. Serial twice dilutions of the chemical compound tested have been added to confluent, 96 well, floor-to-bottom cell monolayers dispensed in the form of a multi-chain pipette (Falcon, NJ, US).

At $37^{\circ} \mathrm{C}$ in an incubator moistened with $5 \% \mathrm{CO}_{2}$ over 24 hours, the microtiter panels were incubated. For each test sample concentration, three wells have been used. Without test sample and DMSO, the control cells were incubated. The small amount of DMSO (maximum $0.1 \%$ ) present in the wells did not affect the experiment. The viable cell yield was determined by a colorimetric method after the incubation at $37^{\circ} \mathrm{C}$ for 24 hours. In brief, after the end of the incubation period, media were aspirated, and the crystal violet solution (1\%) was added to each well for at least 30 minutes.

The stain was removed and the plate was rinsed with tap water to remove all excess stain. Glacial acetic acid (30\%) was then added to all wells and carefully mixed, and the plate absorption was then measured using a test wavelength of $490 \mathrm{~nm}$ after gently shaken micro-plate reader (TECAN, Inc.).
For background absorption found in wells without adding stain all results have been corrected. In the absence of the compounds tested, the treated samples were compared to the cell control. All triplicate experiments have been conducted. Each test compound was calculated for its cell cytotoxic effect. In order to determine the number of sustainable cells, the optical density was measured in the microplate reader (SunRise, TECAN, Inc.) and the viability percentage was measured as:

Viability $=[($ ODt $/$ ODc $)] \times 100 \%$

Where ODt is the mean optical density for tested sample wells and ODc is the average untreated cell optical density. After treatment with the specified compound, the relationship between survival cells and drug concentration is drawn into the survival curve of each tumour cell line. From the graphical plots of the dose response curve for each concentrate with Graphpad Prism software, the $50 \%$ inhibitor concentration $\left(\mathrm{IC}_{50}\right)$, required to cause toxic effects in $50 \%$ of intact cells (San Diego, CA. USA).

\section{Mode of treatment}

$1 \times 10^{6}$ cells/well of HeLa cells were sown in 96 well plates. Resveratrol nanoemulsion (RENE) $(5,10,15,20$ and $25 \mu \mathrm{M})$ was treated 24-hours after incubation. The cells were scraped, washed two times with PBS, lysed for thirty minutes with $520 \mathrm{~mL}$ of potassium chloride $(1,15 \%)$ and centrifuged at fifteen thousand minutes. The methods adapted to Cereser., et al. [25] Ksenija., et al. [26] and Habig and Jacoby [27] have been examined for Lipid Peroxidation, reduced glutathione, and glutathione transfers. According to the Bradford method, the concentration of cell proteins has been determined [28].

On the other hand, tests on pro-inflammatory biomarkers and caspase 3 (units/mg protein) activity were assessed using the proinflammatory Griess reagent at $540 \mathrm{~nm}$ (Protocol) [29], with the support of a platform reader from SpectraMax (Molecular Devices, CA, USA), as described in the manual of the fabricator (ELISA Company Elabscience Biotechnology, Beijing, China). The enzymerelated immuno-sorbent assay (Cayman Chemicals, Ann Arbor, MN, USA) according with the manufacturer's instruction also determined the nuclear factor kappa-B (NF- $\mathrm{B}$ ) p65 subunit DNA binding activity. Three times every experiment was repeated. 


\section{Western blot analysis of P53}

In an assay buffer on radioimmunoprecipitation for $30 \mathrm{~min}$, Hela Cells (human cervical carcinoma) was prepared using the Li., et al. method [30]. In order to remove insoluble material, lysates were centrifuged for $10 \mathrm{~min}$ at 15,000 x g at 4 tube. The protein was collected in the supernatant and maintained for 5 minutes at $95^{\circ} \mathrm{C}$. Protein samples were transferred to polyvinylidene difluoride membranes after 10\% SDS-PAGE gel electrophoresis. The membranes were incubated with anti-p53 (1:3,000) for 1 hour at $37^{\circ} \mathrm{C}$ after blocking 10 percent non-fat milk for 1 hour at room temperature. Three times for 5 minutes, the membranes were washed using BPS. The membranes were incubated for 2 hours at room temperature, by an incubation with an enhanced chemiluminescence detecting reagent, with rabbit $(1: 2,000)$ or goat anti-rabbit antibody (521-31-3; Pierce, Rockford, IL, USA).

\section{Statistical analysis}

Results were expressed as the means \pm Standard Deviation (SD). Differences between groups were evaluated by using one-way ANOVA, followed by Duncan's test. All statistical analyses were performed using the statistical software SPSS 18.0 (SPSS Ltd., Surrey, UK).

\section{Results}

IR spectrum infrared spectrogram of resveratrol nanoemulsion shows a phenol hydroxyl groups absorption peak at $3436 \mathrm{~cm}^{-1}$ as well as benzene ring absorption peaks at 2827, 2920 exists. Light scattering techniques as well as transmission electron microscopy analysis shows that resveratrol nanoemulsion had size of around $49.5 \pm 0.05 \mathrm{~nm}$ (Figure 1) with negative zeta potential of +15.75 .

As shown in the figure 2, the treatment of Hela cells with resveratrol nanoemulsion (RENE) led to a high inhibition in the cell proliferation as concluded by the low $\mathrm{IC}_{50}$ values $(31.89 \mu \mathrm{g} / \mathrm{ml})$ which revealed a high anti-tumor activity of the RENE against human cervical carcinoma.

Figure 3 showed the induction effect of RENE on TBARS in Hela cells. As shown, TBARS was dramatically increased after RENA treatment at (5 - $25 \mu \mathrm{M})$. Moreover, RENA (5 - $25 \mu \mathrm{M})$ treatment also inhibit the level of GSH and activity of GST (Figure 4 and 5).

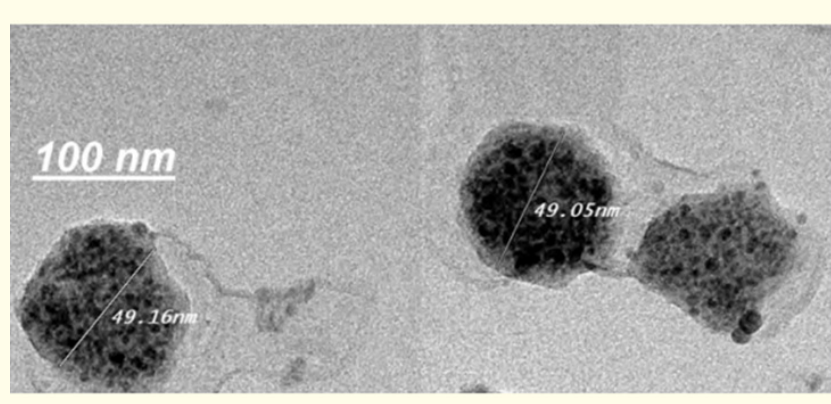

Figure 1: Morphology and size measurements of resveratrol nanoemulsion characterized by TEM are shown, with scale bar $100 \mathrm{~nm}$, respectively.

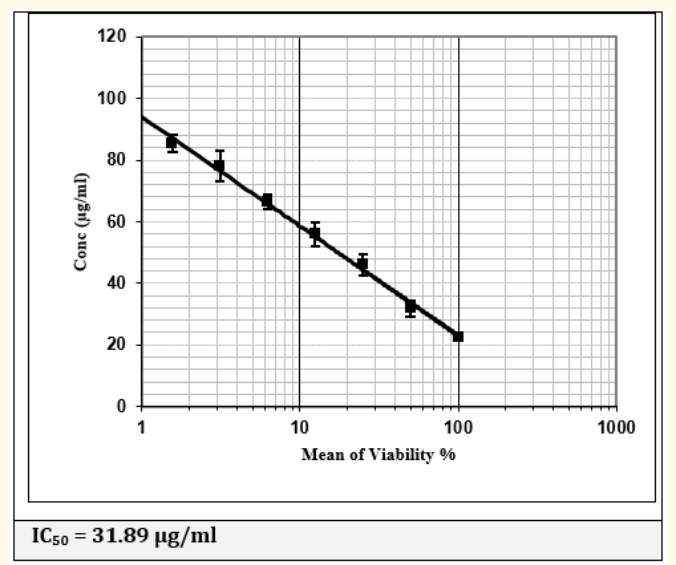

Figure 2: $\mathrm{IC}_{50}(\%)$ of resveratrol nanoemulsion against Hela cells after $24 \mathrm{~h}$ of incubation as assayed by MTT $(\mathrm{n}=4)$.

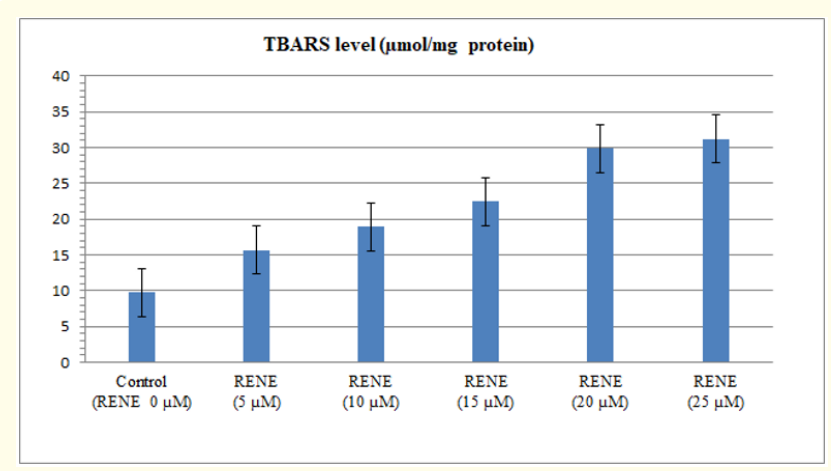

Figure 3: The effect of treatment with resveratrol nanoemulsion (RENE) (5-25 uM), on TBARS levels in Hela cells. 


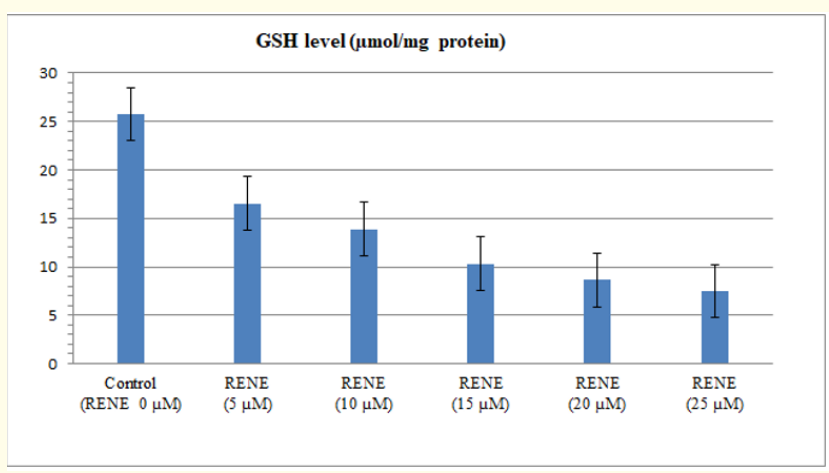

Figure 4: The effect of treatment with resveratrol nanoemulsion (RENE) (5-25 uM), on reduced glutathione (GSH) levels in Hela cells.

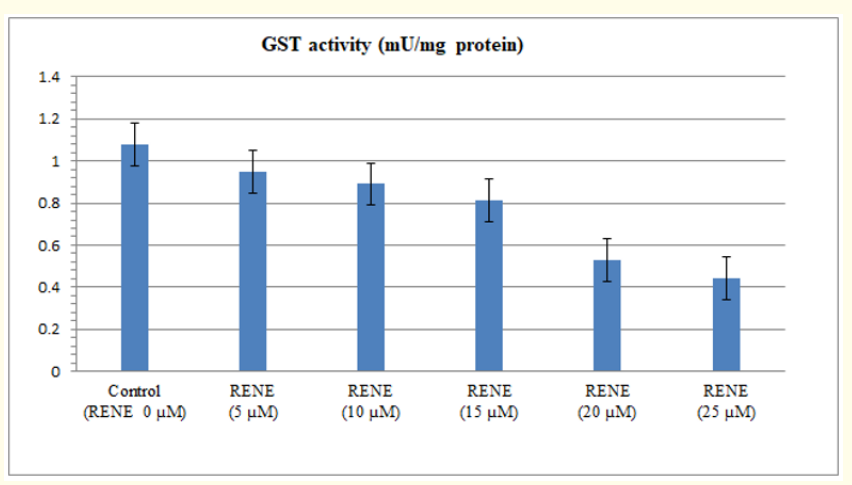

Figure 5: The effect of treatment with resveratrol nanoemulsion (RENE) (5-25 uM), on glutathione $\mathrm{S}$ transferase (GST) activity in Hela cells.

The results in figure 6 illustrated that RENA at $(5-25 \mu \mathrm{M})$ produced a marked elevation of caspase activity. Taken together, these results suggested that RENE displayed rapid and potent anti-tumor effects against Hela cell lines. Also, the present study further detected the inhibitory effect of RENA at (5 - $25 \mu \mathrm{M})$ on NF- $\mathrm{KB}$ activity in Hela cells (Figure 7). The expression of p53 protein was increased significantly in the RENA at ( 5 - $25 \mu \mathrm{M})$ treated HeLa cells compared with that in the control group (Figure 8 and 9).

\section{Discussion}

Resveratrol is a promising natural compound for many diseases prevention and treatment. However, its low levels of stability and cellular bioavailability limit its anti-tumor activity. Two approaches have been used to increase its stability and bioactivities: (i) forma-

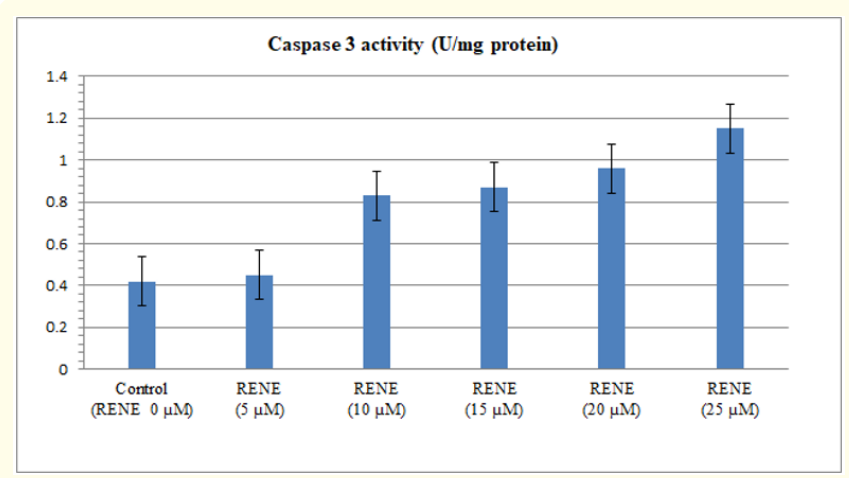

Figure 6: The effect of treatment with resveratrol nanoemulsion (RENE) (5-25 uM), on caspase activity in Hela cells.

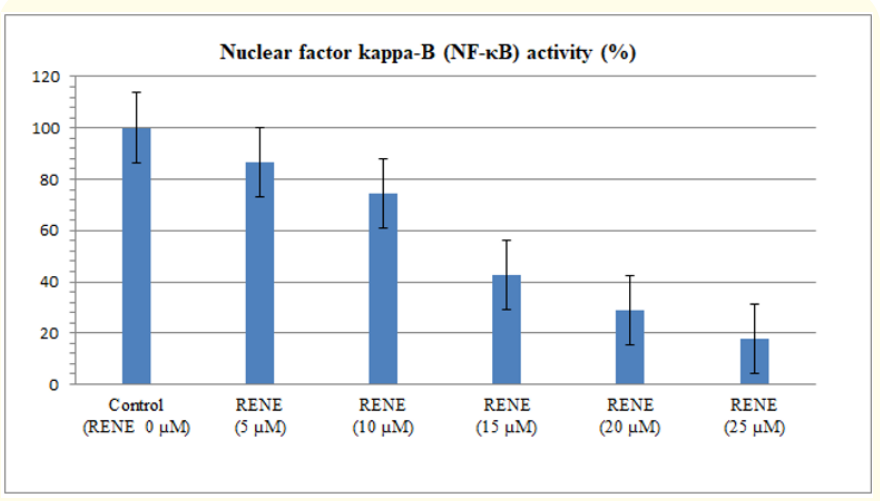

Figure 7: The effect of treatment with resveratrol nanoemulsion (RENE) (5-25 uM), on Nuclear factor kappa-B (NF- $\kappa$ B) activity in Hela cells.

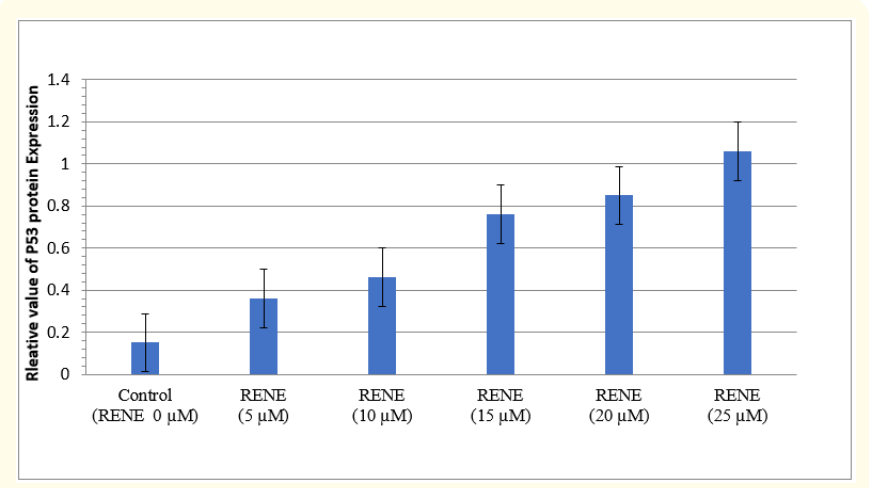

Figure 8: The effect of treatment with resveratrol nanoemulsion (RENE) (5-25 uM) on relative expression value of P53 protein in Hela cells. 


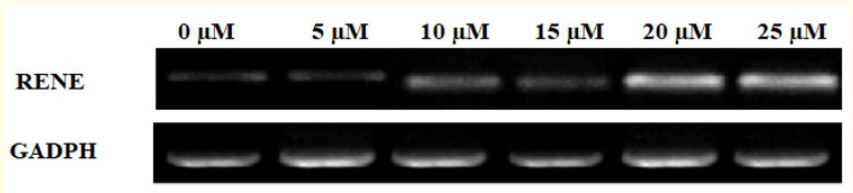

Figure 9: The effect of treatment with resveratrol nanoemulsion (RENE) (5-25 uM) on electrophoretic pattern of P53 protein in Hela cells.

tion peracetate ester of resveratrol [31] or resveratrol -docosapentaenoic acid ester [32]; (ii) using nanocarriers such as nanoliposomes [33] and NLCs [34]. The chemical modification makes lipophilic resveratrol prodrugs, which require chemical cleavage before releasing nonencapsulated resveratrol. Nanoliposomes are not stable, and encapsulated compound can be leaked out. NLCs do not have those problems and have been widely used in pharmaceutical and nutraceutical research. There are several characteristics necessary for a chitosan to exhibit ionic interaction with resveratrol. These include:

- $\quad$ Strong hydrogen-bonding groups (-OH, $-\mathrm{COOH})$.

- High molecular weight

- $\quad$ Sufficient chain flexibility

- $\quad$ Surface energy properties favoring spreading onto mucus. Also, the presence of lone pairs of electrons in hydroxyl groups of resveratrol facilities the formation of hydrogen bonds with amino groups of chitosan (Figure 10).

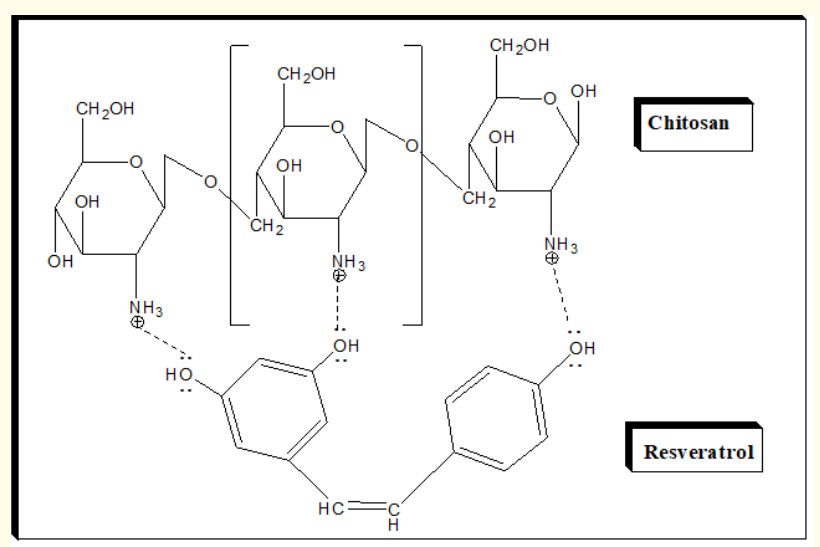

Figure 10: Proposal diagram for interaction between chitosan and resveratrol.
Infrared spectrogram of the resveratrol raw powder shows a phenol hydroxyl group absorption peak at $3252 \mathrm{~cm}^{-1}$ and benzene ring absorption peaks at 2827, 2920 exists. Infrared spectrogram of RENE shows a hydroxyl group absorption peak at $3436 \mathrm{~cm}^{-1}$ exists, but the characteristic absorption peak of resveratrol did not appear, proving that resveratrol had been completely wrapped in chitosan.

Our results in confirmed with the results of Hussein., et al. [17] showed that the addition of chitosan in presence tripolyphosphate will lead to increase particle diameters.

To further determine the size of the RENE in the dry state, electron microscopic analysis was performed. TEM images illustrated both the size and morphology of plain NLC and CSNLC (Figure 1).

At $\mathrm{pH} 7, \mathrm{NH}_{3}{ }^{+}$of chitosan get deprotonated and changing to $\mathrm{NH}_{2}$ negatively group. Also, hydroxyl group of resveratrol which is negatively charged is no longer able to interact with $\mathrm{NH}_{2}$ group of chitosan and leading to the release of resveratrol from nanoparticles.

\section{Anti-tumor activity}

Using MTT assay, the effect of the resveratrol encapsulated (NLC) and resveratrol-chitosan coated (CSNLC) on the proliferation of Hela cells was studied after $48 \mathrm{~h}$ of incubation.

This study demonstrated that RENE was decrease the viability of Hela cells. From research in the field of nanotechnology, it was noted that nanoparticles improved drug solubility, controlled drug release, enhanced bioavailability, increased stability and improved long-term storage [35]. Resveratrol possesses antiproliferative effects through the induction of death in many different cell lines, including colon cancer [36]. Also, it has low solubility, stability, and bioavailability [37]. Nanoparticles were shown to improve the stability and enhancing compound's bioavailability [38]. The results of the MTT assay with nanoparticles indicate the anticancer activity of both resveratrol formulas. This may be due to the resveratrol encapsulated nanoparticles' rate of bioavailability. Many studies indicate that nanoparticles improve bioavailability [39]. However, if the rate of absorption is too great, it may lead to toxicity on cancer cells. This could be the issue in the current study. 
Cells withstand and counteract oxidative stress using several and different defense mechanisms, ranging from free radical scavengers and antioxidant molecules/enzymes to sophisticated and elaborate DNA repair mechanisms to reduce excessive levels of ROS and prevent irreversible cellular damage $[40,41]$. An increased rate of ROS production occurs in highly proliferative cancer cells, owing to the presence of oncogenic mutations that promote aberrant metabolism. Increased oxidative stress is well documented in transformed cells [42-45] and growing evidence suggests that ROS act as second messengers in intracellular signaling cascades which induce and maintain the oncogenic phenotype of cancer cells [46]. During cervical carcinogenesis an increase of oxidative DNA damage, as shown by progressive increase in the levels of 8-OHdG from normal to SIL to invasive carcinomas, has been reported [47]. In our recent work we demonstrated that RENE-induced oxidative stress and decrease the GSH level and GST activity which could be provoked an increased and selective protein oxidation in keratinocyte cells transfected with the whole HPV-16 genome [48].

Apoptosis can be started through two distinct pathways, the death receptor (extrinsic pathway) mitochondrial mediated (intrinsic pathway). Both lead to activation of the caspase cascades "executioner caspases" such as caspase-3, -6 and -7. The active executioner caspases bind cellular substrates, leading to the characteristic biochemical and morphological changes. This is followed by chromatin condensation, nuclear shrinkage and DNA fragmentation. Binding of cytoskeletal proteins leads to cell fragmentation and formation of apoptotic bodies $[17,49,50]$. As shown in the present results caspase- 3 were significantly increased in Hela cells treated with the RENE compared to untreated cells. Many research studies have demonstrated that resveratrol can induce cancer cell death in cellular studies [51-53]. This is a promising attribute in the field of cancer research. It is important for the area of nanoparticles to perfect precise targeting of cancer cells, and optimal time release.

In regard of resveratrol antitumoral properties, several studies were conducted on certain cancer cell lines [17]. Cervical cancer cell lines were often used as a preferred carcinogenic model to understand molecular targets and mechanism of resveratrol action. Here are reported all the in vitro advances for the use of RENE in the treatment of cervical cancer. In the present study we explain the effect of RENE on NF- $\mathrm{B}$ activity and P53 protein expression in cervical cancer cells. we demonstrated that inhibition of NF- $\kappa B$ activity and activation of P53 protein expression induced by RENE leading to increase cell-death. According with this study Bava., et al. [54] showed that other polyphenolic compounds sensitizes cervical cancer cells to the therapeutic effect of taxol, acting in the down-regulation of both NF- $\kappa \mathrm{B}$ and serine/threonine kinase AKT pathway, a survival signal related to NF- $\mathrm{BB}$.

Prevention of DNA oxidation is also achieved by these polyphenols mainly by quenching free radicals and modulating bio-metabolism enzymes [13]. This study demonstrated that RENE was more potent anticancer agent against Hela cell line due to the nanoparticles improved drug solubility, controlled drug release, enhanced bioavailability, increased stability and improved long-term storage [17]. This study, indicate that NF- $\kappa B$, is one of the central players in the synergism of RENE and act as a regulator of caspase-3 and, P53 instead is the regulator of NF- $\kappa B$, which through the phosphorylation of MAPKs regulates a set of survival signals.

\section{Conclusion}

In the present study, we used the chitosan to prepare resveratrol nanoemulsion. Also, characterization of obtained spherical nanoparticles was evaluated using different procedures. RENE showed significant anticancer activity against Hela cells line. Also, RENE possess significant apoptotic properties via inhibition of GSH, GST and NF- $\mathrm{B}$ as well as activation of the level of TBARS, caspase-3 activity and $\mathrm{P} 53$ protein expression. Overall, our results suggested that RENE could be a promising new approach for anticancer therapies.

\section{Ethics Approval and Consent to Participate}

Ethical approval for the data collection was granted by the Research Ethics Committee at the Faculty of Applied Medical Sciences, October 6 University, Egypt (No. 20201206). No lab. animals or Humans were used studies that are base of this research, in-vitro experiments only were used.

\section{Consent for Publication}

The authors gave consent for their data to be used in the article. 


\section{Availability of Data and Materials}

Supporting data will be made available as it contains spectroscopic and analytical data to prove the structure of obtained nanoparticles as well as their in vitro antitumor and antiapoptotic activities.

\section{Competing Interests}

The authors declare no conflict of interest, financial or otherwise.

\section{Funding}

The authors are not currently in receipt of any research funding.

\section{Bibliography}

1. Siegel RL., et al. "Cancer statistics, 2017”. CA: A Cancer Journal for Clinicians 67.1 (2017): 07-30.

2. Narayana G., et al. "Knowledge, attitude, and practice toward cervical cancer among women attending Obstetrics and Gynecology Department: A cross sectional, hospital-based survey in South India". Indian Journal of Cancer 54 (2017): 481-487.

3. Baindara P., et al. "Anticancer properties of a defensin like class IIdbacteriocin Laterosporulin10". Scientific Report 7 (2017): 46541.

4. Kelloff GJ., et al. "Progress in cancer chemoprvention: development of diet-derived chemopreventive agents". Journal of $\mathrm{Nu}$ trition 130 (2000): 467S-471S.

5. Masters JR. "Human cancer cell lines: fact and fantasy". Nature Reviews Molecular Cell Biology 1.3 (2000): 233-236.

6. El-gizawy HA and Hussein MA. "Fatty acids Profile, Nutritional Values, Anti-diabetic and Antioxidant Activity of the Fixed Oil of Malva parviflora Growing in Egypt". International Journal of Phytomedicine 7 (2015): 219-230.

7. Seeram N., et al. "Sources and chemistry of resveratrol". Resveratrol health and disease. CRC Press, Boca Raton, FL, USA (2006): 17-32.

8. Hung LM., et al. "Cardioprotective effect of resveratrol, a natural antioxidant derived from grapes". Cardiovascular Research 47 (2000): 549-555.
9. Das S., et al. "Coordinated induction of iNOS-VEGF-KDR-eNOS after resveratrol consumption: a potential mechanism for resveratrol preconditioning of the heart". Vascular Pharmacology 42 (2005): 281-289.

10. Atten MJ., et al. "Resveratrol-induced inactivation of human gastric adenocarcinoma cells through a protein kinase Cmediated mechanism". Biochemical Pharmacology 62 (2001): 1423-1432.

11. El-Mowafy AM and Alkhalaf M. "Resveratrol activates adenylyl-cyclase in human breast cancer cells: a novel, estrogen receptor-independent cytostatic mechanism". Carcinogenesis 24 (2003): 869-873.

12. de la Lastra CA and Villegas I. "Resveratrol as an antioxidant and pro-oxidant agent: mechanisms and clinical implications". Biochemical Society Transactions 35 (2007): 1156-1160.

13. Hussein MA. "A Convenient Mechanism for the Free Radical Scavenging Activity of Resveratrol". International Journal of Phytomedicine 3 (2011): 459-469.

14. El-gizawy A., et al. "Chemical Composition of the Essential oil of the Leaves of Pimenta diocia (L.) Merr. and Pimenta racemosa (Mill.) cultivated in Egypt and evaluation of their in-vitro antioxidant and antidiabetic". International Journal of Phytomedicine 4 (2018): 226-234.

15. Vasanthi HR., et al. "Phytochemicals from plants to combat cardiovascular disease". Current Medicinal Chemistry 19 (2012): 2242-2251.

16. Yang Q., et al. "Resveratrol inhibits the growth of gastric cancer by inducing g1 phase arrest and senescence in a sirt1-dependent manner". PLoS One 8 (2013): e70627.

17. Hussein MA., et al. "Resveratrol Nanoemulsion: A Promising Protector Against Ethinylestradiol-Induced Hepatic Cholestasis in Female Rats". Journal of Biomolecular Research and Therapeutics 8 (2020): 1-7.

18. Mahmoud RA., et al. "Resveratrol Nanoemulsion A Promising regulator of TGFB1and TFF-3 Genes Expression in DSSInduced Ulcerative Colitis in Rats". Acta Scientific Nutritional Health 5 (2021): 18-29. 
19. Mohamed FT., et al. "Resveratrol Nanoemulsion A Promising Inhibitor against Mitogen-Activated Protein Kinase-Dependent Inflammation and Ameliorates Nicotine Induced-lung Toxicity in Rats". Free Radical and Antioxidants 10.1 (2020): $35-41$.

20. Hussein MA and Abdel-Gawad SA. "In vivo Hepatoprotective Properties of Purslane Extracts on Paracetamol-Induced Liver Damage". Malaysian Journal of Nutrition 16.1 (2010): 160-170.

21. El-gizawy HA and Hussein MA. "Fatty acids Profile, Nutritional Values, Anti-diabetic and Antioxidant Activity of the Fixed Oil of Malva parviflora Growing in Egypt". International Journal of Phytomedicine 7 (2015): 219-230.

22. Hussein MA. "Cardioprotective Effects of Astaxanthin against Isoproterenol-Induced Cardiotoxicity in Rats". Journal of $\mathrm{Nu}$ trition and Food Sciences 5 (2017): 335.

23. Hussein MA and Boshra SA. "Antitumor and structure antioxidant activity relationship of colchicine on Ehrlich ascites carcinoma (EAC) in female mice". International Journal of Drug Delivery 5 (2013): 430-437.

24. Hussein MA. "Anti-inflammatory effect of natural heterocycle glucoside vicine obtained from Vicia faba L. and its aglucone (divicine) and their effect on some oxidative stress biomarkers in Albino rats". Free Radicals and Antioxidants 2 (2012): 44-55.

25. Cereser CS., et al. "Thiram-induced cytotoxicity is accompanied by a rapid and drastic oxidation of reduced glutathione with consecutive lipid peroxidation and cell death". Toxicology 163 (2001): 153-162.

26. Ksenija D., et al. "Cytotoxic and Apoptotic Effect of Structurally Similar Flavonoids on Parental and Drug-Resistant Cells of a Human Cervical Carcinoma". Food Technology and Biotechnology 47 (2009): 356-363.

27. Habig WH and Jacoby WB. "Assays for determination of glutathione S-transferases". Methods Enzymology 77 (1981): 398405 .

28. Bradford MM. "A rapid and sensitive method for the quantification of microgram quantities of protein utilising the principle of protein-dye binding". Annals of Biochemistry 72 (1976): 248-254.
29. Green LC., et al. "Analysis of nitrate, nitrite and [15N]nitrate in biological fluids". Annals of Biochemistry 162 (1982): 131-138.

30. Li GL., et al. "HPV E6 down-regulation and apoptosis induction of human cervical cancer cells by a novel lipid-soluble extract (PE) from Pinellia pedatisecta Schott in vitro". Journal of Ethnopharmacology 132 (2010): 56-64.

31. Lam WH., et al. "A potential prodrug for a green tea polyphenol proteasome inhibitor: evaluation of the peracetate ester of (-)-epigallocatechin gallate [ (-)-EGCG]". Bioorganic and Medicinal Chemistry 12.21 (2004): 5587-5593.

32. Zhong Y., et al. "Anti-inflammatory activity of lipophilic epigallocatechin gallate (EGCG) derivatives in LPS-stimulated murine macrophages". Food Chemistry 134.2 (2012): 742-748.

33. de Pace RC., et al. "Anticancer activities of (-)-epigallocatechin3-gallate encapsulated nanoliposomes in MCF7 breast cancer cells". Journal of Liposome Research 23.3 (2013): 187-196.

34. Barras A., et al. "Formulation and characterization of polyphenol-loaded lipid nanocapsules". International Journal of Pharmaceutics 379.2 (2009): 270-277.

35. Ibrahim WM., et al. "Novel sulpiride-loaded solid lipid nanoparticles with enhanced intestinal permeability". International Journal of Nanomedicine 9 (2014): 129-144.

36. Aggarwal BB., et al. "Role of resveratrol in prevention and therapy of cancer: preclinical and clinical studies". Anticancer Research 24 (2004): 2783-2840.

37. Cottart $\mathrm{CH}$., et al. "Resveratrol bioavailability and toxicity in humans". Molecular Nutrition and Food Research 54 (2010): 7-16.

38. Constantinides PP., et al. "Advances in lipid nanodispersions for parenteral drug delivery and targeting". Advanced Drug Delivery Reviews 60 (2008): 757-767.

39. Wang Y., et al. "Effect of staurosporine on the mobility and invasiveness of lung adenocarcinoma A549 cells: an in vitro study". BMC Cancer 9 (2009): 174.

40. Mates J., et al. "Antioxidant enzymes and human diseases". Clinical Biochemistry 32 (1999): 595-603. 
41. Maynard S., et al. "Base excision repair of oxidative DNA damage and association with cancer and aging". Carcinogenesis 30 (2009): 2-10.

42. Storz P. "Reactive oxygen species in tumor progression". Frontiers in Bioscience 10 (2005): 1881-1896.

43. Burdon RH. "Superoxide and hydrogen peroxide in relation to mammalian cell proliferation". Free Radical Biology and Medicine 18 (1995): 775-794.

44. Toyokuni S., et al. "Persistent oxidative stress in cancer". FEBS Letter 358 (1995): 1-3.

45. Kryston TB., et al. "Role of oxidative stress and DNA damage in human carcinogenesis". Mutation Research 711 (2011): 193201.

46. Liou GY and Storz P. "Reactive oxygen species in cancer". Free Radical Research 44 (2010): 479-496.

47. Sgambato A., et al. "Decreased expression of the CDK inhibitor p27Kip1 and increased oxidative DNA damage in the multistep process of cervical carcinogenesis". Gynecologic Oncology 92 (2004): 776-783.

48. Perluigi M., et al. "Proteomics analysis of protein expression and specific protein oxidation in human papillomavirus transformed keratinocytes upon UVB irradiation". Journal of Cellular and Molecular Medicine 13 (2009): 1809-1822.

49. Estaquier J., et al. "The mitochondrial pathways of apoptosis". Advances in Experimental Medicine and Biology 942 (2012): 157-183.

50. Krysko DV and Vandenabeele P. "From regulation of dying cell engulfment to development of anti-cancer therapy". Cell Death and Differentiation 15 (2008): 29-38.

51. Opipari AW., et al. "Resveratrol-induced autophagocytosis in ovarian cancer cells". Cancer Research 64 (2004): 696-703.

52. Park JW., et al. "Resveratrol induces pro-apoptotic endoplasmic reticulum stress in human colon cancer cells". Oncology Report 18 (2007): 1269-1273.
53. Yang Q., et al. "Resveratrol inhibits the growth of gastric cancer by inducing $\mathrm{g} 1$ phase arrest and senescence in a sirt1-dependent manner". PLoS One 8 (2013): e70627.

54. Nair S., et al. "Pharmacogenomics of phenolic antioxidant butylated hydroxyanisole (BHA) in the small intestine and liver of Nrf2 knockout and C57BL/6J mice". Pharmaceutical Research 23 (2006): 2621-2637.

\section{Assets from publication with us}

- Prompt Acknowledgement after receiving the article

- Thorough Double blinded peer review

- Rapid Publication

- Issue of Publication Certificate

- High visibility of your Published work

Website: www.actascientific.com/

Submit Article: www.actascientific.com/submission.php

Email us: editor@actascientific.com

Contact us: +919182824667 